\title{
Determination of temporal distribution of moment release using long period body wave data: the case of the 2003 Tokachi-Oki earthquake
}

\author{
Tatsuhiko Hara \\ International Institute of Seismology and Earthquake Engineering, Building Research Institute, Tatehara 1, Tsukuba, Ibaraki 305-0802, Japan
}

(Received December 4, 2003; Revised February 1, 2004; Accepted February 23, 2004)

\begin{abstract}
We performed waveform inversion of long period body wave data to investigate whether it is possible to determine the moment release distribution in time and space, using the 2003 Tokachi-Oki earthquake as an example. Our results show that it is difficult to determine the precise spatial distribution, and therefore we focused on determination of the temporal distribution. We allowed changes of the source mechanism during rupture in the inversion scheme. The temporal change of the scalar moment of the subevents was consistent with the moment rate function inferred from other studies. The source mechanisms of the subevents were primarily reverse faults, matching preliminary reports of this earthquake. No significant change of the source mechanism during the rupture were inferred. Our results suggest that the rupture process of this earthquake was rather simple.
\end{abstract}

Key words: Centroid moment tensor, temporal distribution, long period body wave.

\section{Introduction}

More than twenty years have passed since the Harvard University group started determination of CMT solutions on a routine basis (Dziewonski and Woodhouse, 1983). Considering the expansion of broadband seismic networks in the last two decades, it may be possible to routinely determine a larger number of earthquake source parameters than nine parameters of CMT (five for the moment tensor under the condition that its trace is zero, three for the centroid location and the centroid time). As such an attempt, we performed waveform inversion of long period body wave data to investigate whether it is possible to determine spacetime distribution of the moment release of the September 25, 2003 Tokachi-Oki earthquake (origin time: 19:50:06 UTC, September 26, 04:50:06 local time at epicenter; location: $41.78^{\circ} \mathrm{N} 143.86^{\circ} \mathrm{E}$; depth: $27 \mathrm{~km}$; Mw: 8.1 after USGS).

\section{Analysis}

We assumed the source region of the 2003 Tokachi-Oki earthquake based on the available information before we started our analysis with the hypocenter, magnitude, and rupture models (i.e., Yagi, 2003). Figure 1(a) shows the assumed source region in this study. We put the $5 \times 5$ grids horizontally (Fig. 1(a)), and 3 grids vertically at depths of 20,30 and $40 \mathrm{~km}$. Assuming that the rupture process time is 60 seconds, we put 5 grids in time with the constant interval (i.e., the time interval between the adjacent grids is 15 seconds). We calculated the Green's function for each moment tensor component for each pair of the space and time grids using the Direct Solution Method (Cummins et al., 1994; Takeuchi and Geller, 1996). We used PREM

Copy right(c) The Society of Geomagnetism and Earth, Planetary and Space Science (SGEPSS); The Seismological Society of Japan; The Volcanological Society of Japan; The Geodetic Society of Japan; The Japanese Society for Planetary Sciences; TERRAPUB
(Dziewonski and Anderson, 1981) as an earth model for which the Green's functions were computed.

We retrieved long period channel data from IRIS DMC. The number of seismograms was 69 . We did not set the distance range for data selection. We edited the parts of seismograms between the beginnings of the records and the arrivals of surface waves, calculated the Fourier spectra, corrected the instrumental response, and employed the spectra in the frequency band between $0.01-0.02 \mathrm{~Hz}$ as dataset of the present study.

First, in order to investigate the resolving power of the current dataset for space distribution of the moment release, we performed inversion for moment tensor using the Green's function computed for each pair of the space and time grids. That is, the number of model parameters is five under the condition that the trace of the moment tensor is zero, and we performed inversion 375 times ( 25 horizontal grids $\times 3$ vertical grids $\times 5$ time grids). Figures 1(b) and (c) show a part of the results obtained using the Green's function computed for sources at a depth of $30 \mathrm{~km}$ for a time grid 30 seconds after the origin time. We have determined the largest variance reduction for this depth and time grid. Similar large variance reductions were obtained for many grids. The source mechanisms determined for those grids are similar to each other. This suggests that the resolution of the current dataset for spatial distribution was poor, and that it is difficult to obtain a precise spatial distribution. Therefore, we fixed the centroid location in the following analysis to determine the temporal distribution of the moment release. Based on the results shown above together with the rupture model (Yagi, 2003), we put the centroid on $42^{\circ} \mathrm{N}, 144^{\circ} \mathrm{E}$.

We assumed that synthetic seismograms are expressed by summation of the product of the moment tensor component at each time grid and its corresponding Green's function and performed waveform inversion of observed seismograms to determine the moment tensor at each time grid simultane- 


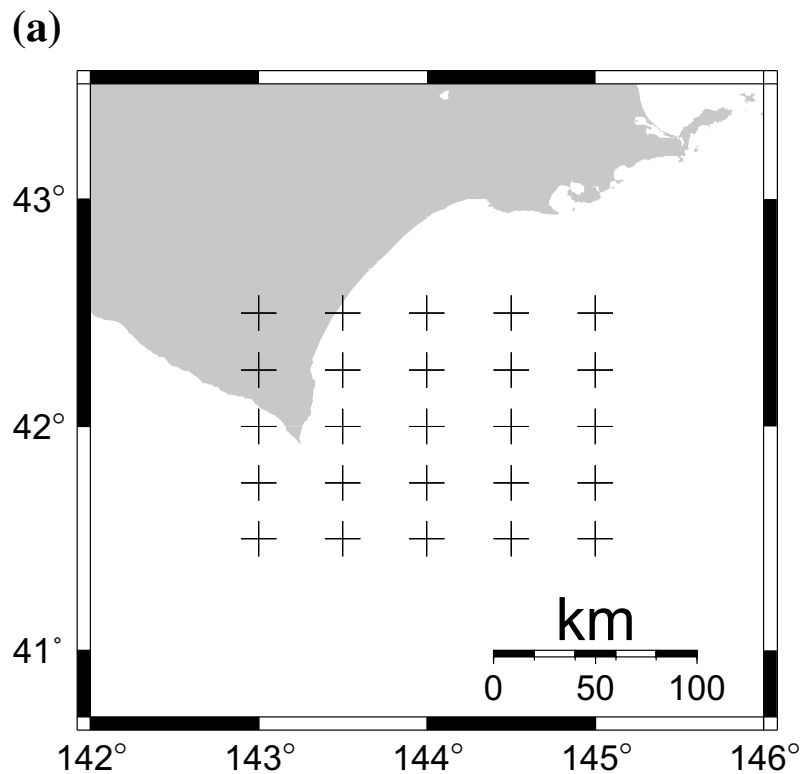

(b)

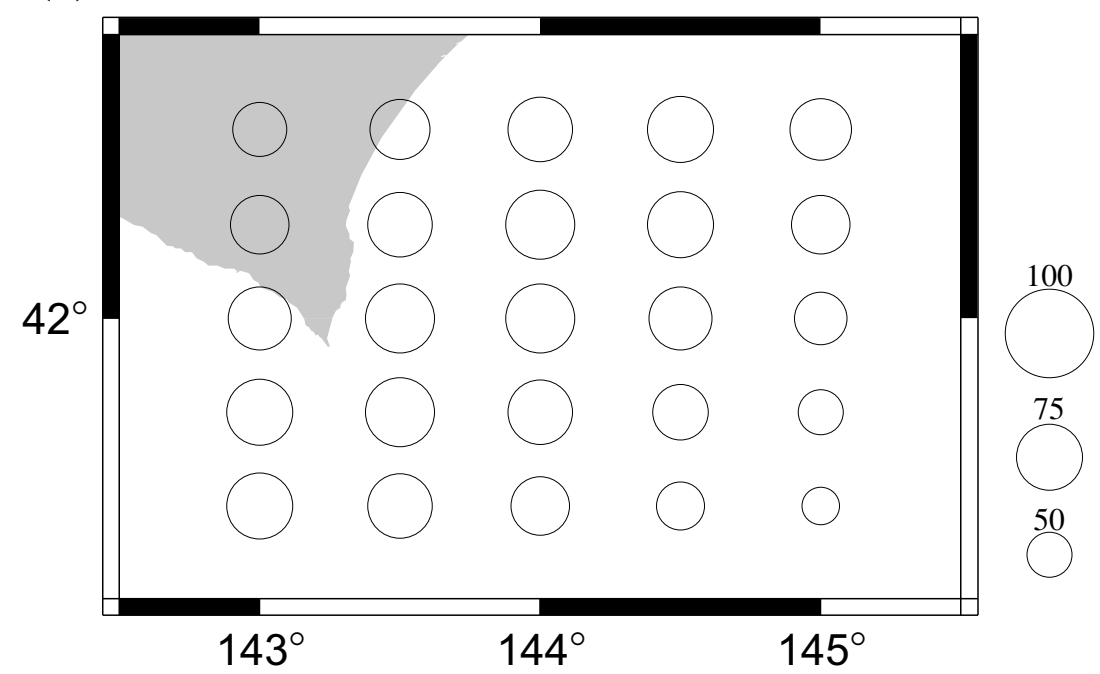

(c)

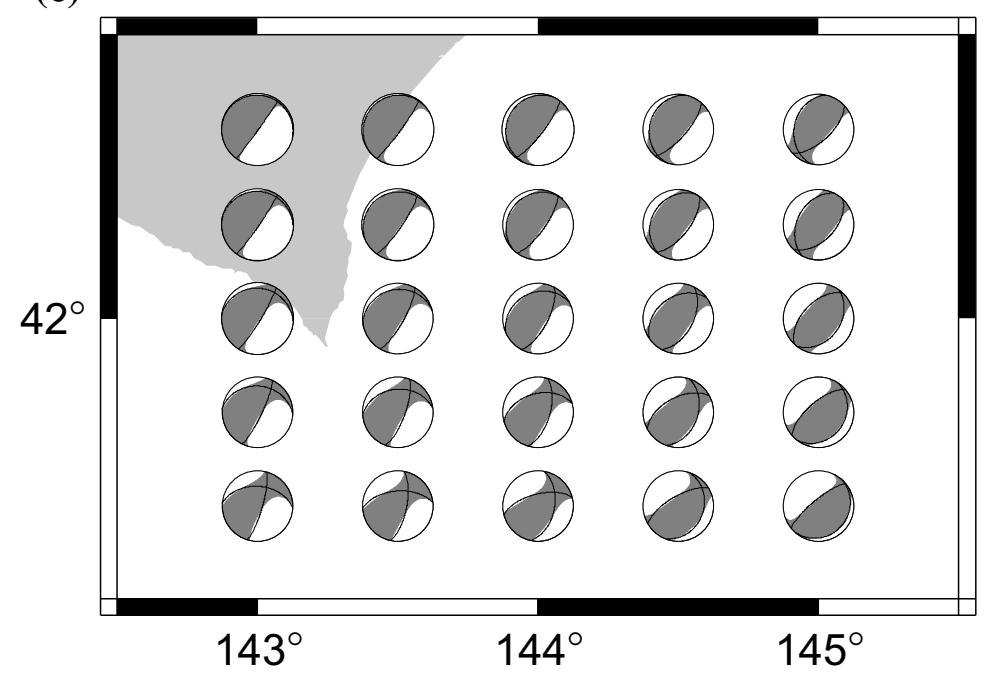

Fig. 1. (a) The source region assumed in this study. The plus signs denote the horizontal grids. (b) The variance reduction obtained by inversion using the Green's functions computed for each grid at a depth of $30 \mathrm{~km}$ for a time grid 30 seconds after the origin time. The diameters of circles are proportional to the variance reduction. The scales are shown in the right-hand side of the figure. (c) The obtained source mechanisms. 


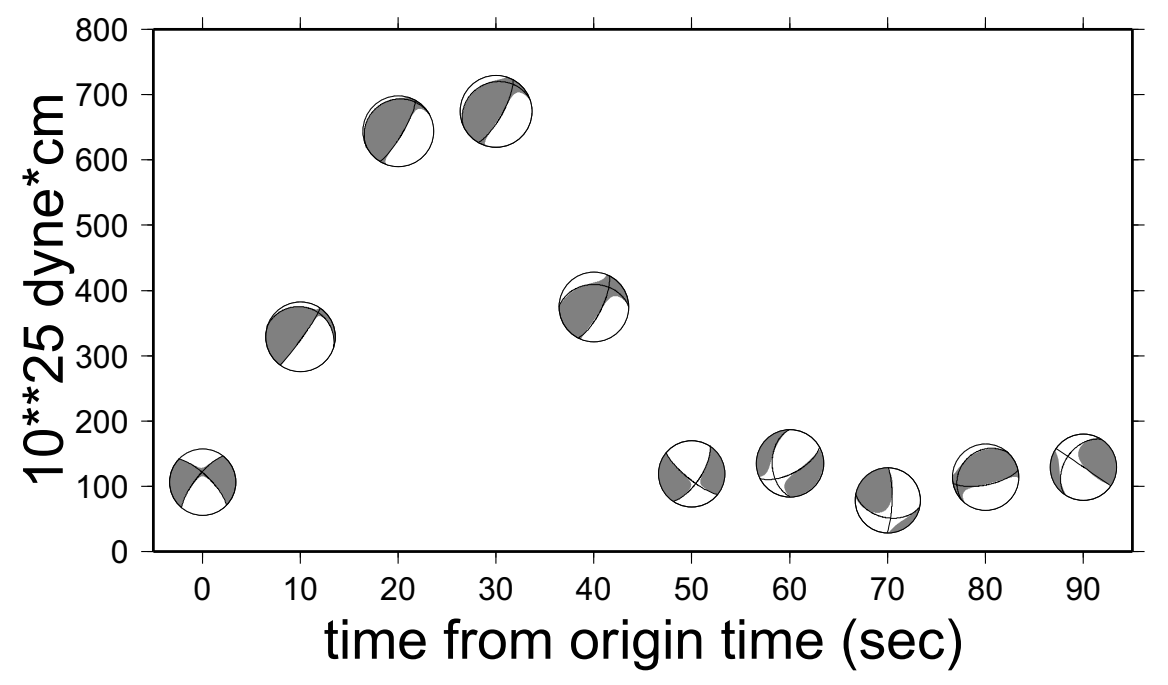

Fig. 2. The temporal distribution of the moment release for the 2003 Tokachi-oki earthquake. The vertical axis shows the scalar moments of subevents.

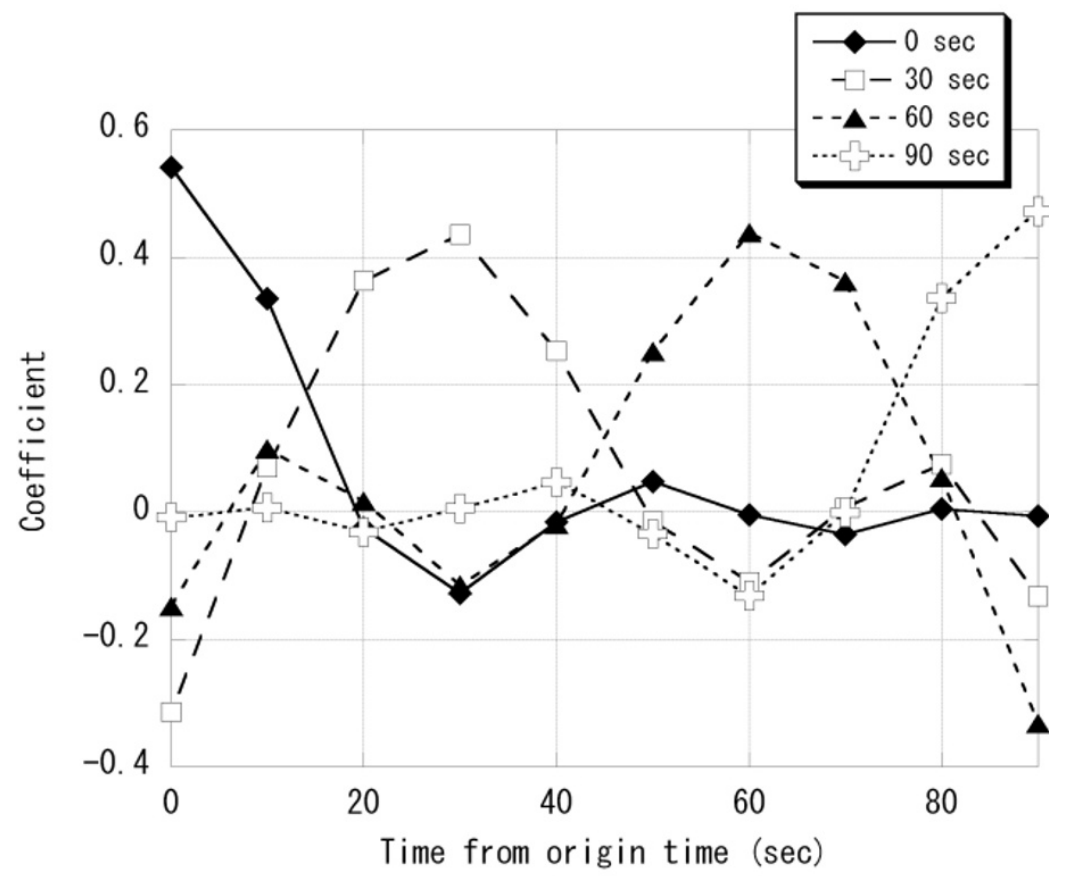

Fig. 3. The resolution matrix coefficients for Mrr. The diamonds, squares, triangles, and crosses denote the coefficients between 0 sec and $0-90$ sec, 30 sec and 0-90 sec, $60 \mathrm{sec}$ and $0-90 \mathrm{sec}, 90 \mathrm{sec}$ and $0-90 \mathrm{sec}$, respectively.

ously. This inverse problem is linear and the temporal change of the source mechanisms is allowed. In order to determine the precise temporal distribution, we lengthened the assumed rupture duration to be 90 seconds, and put 10 grids with the constant interval (i.e., the time interval between the adjacent grids is 10 seconds). The number of the unknowns was 50, since we assumed that the trace of the moment tensor was zero ( 5 moment tensor components $\times 10$ time grids). We adopted the smoothness constraint that enforced the smoothness between the same moment tensor components on the adjacent time grids. We solved the following equation:

$$
\left(\mathbf{G}^{T} \mathbf{G}+\alpha^{2} \mathbf{S}\right) \mathbf{m}=\mathbf{G}^{T} \mathbf{d}
$$

where $\mathbf{G}$ is the kernel matrix consisting of the Green's functions, $\mathbf{S}$ is the smoothness matrix, $\alpha^{2}$ is the weight of the smoothness constraint, $\mathbf{m}$ is the model vector consisting of moment tensor components of subevents, and $\mathbf{d}$ is the data vector. $T$ denotes the transpose of matrices. We varied the value of $\alpha^{2}$ to find a solution that is consistent with the preliminary CMT solution by Hara (2003) in that scalar moments of subevents are of the same order of the scalar moment of the CMT solution, and in that the scalar moment of the sum of subevents are comparable to that of the CMT solution. We found such consistent solutions for a range of $\alpha^{2}=10^{-6}-10^{-7}$ and those solutions were qualitatively similar to each other.

Figure 2 shows the solution obtained for $\alpha^{2}=10^{-7}$. The large moment release was found 20-30 seconds after the origin time, and the main rupture duration was about 40 seconds. This characteristics are similar to the 
moment rate function inferred from teleseismic broadband $P$ and $S H$ waves (e.g., Yagi, 2003; Yamanaka and Kikuchi, 2003). The source mechanisms of the subevents during the first 40 seconds were primarily reverse faults, which is consistent with preliminary source mechanism solutions of this earthquake such as Harvard CMT (http://www.seismology.harvard.edu/CMTsearch.html) and USGS Fast moment tensor solution (http://wwwneic.cr. usgs.gov/neis/eq_depot/2003/eq_030925/neic_zdap_q.html). Although the source mechanisms of the subevents at $0 \mathrm{sec}$ and during 50-90 sec were different from those during $10-40 \mathrm{sec}$, the absolute values of their estimates were around 2 times of their standard deviations. Therefore, it is difficult to conclude that there was a significant change of source mechanism during the rupture.

Figure 3 illustrates the temporal resolution. Since the coefficients of the resolution matrix between the different moment tensor components are negligible, we showed the coefficients for Mrr. The resolution for the other components was similar to that for Mrr. The temporal resolution was about 20 sec, and it was possible to detect a change of source mechanism if its time scale was around or larger than this resolution. Therefore, in the present study, no significant change of the source mechanism whose time scale was around or larger than $20 \mathrm{sec}$ was inferred. For the time grids of 30 and $60 \mathrm{sec}$ (squares and triangles in Fig. 3), the large negative coefficients were obtained at 0 and $90 \mathrm{sec}$, respectively. These coefficients were not likely to affect solutions considerably, because the moment release before and after the assumed rupture process time was set to zero, and because the scalar moments of subsvents at the starting and ending time grids were likely to be relatively small due to the smoothness constraint.

\section{Discussion}

We have shown that it is possible to obtain a reliable temporal distribution of moment release using long period body wave data. Considering its simple data processing and inversion scheme, it is likely to be easy to apply the technique of the present study to analyses of large earthquakes on a routine basis. Although there have been many previous studies that determined temporal distribution of moment release for large earthquakes (e.g., Ruff and Miller, 1994; Riedesel et al., 1986), there are few attempts for routine determination. The project of the University of Michigan group is an attempt in this direction (Tanioka and Ruff, 1997). Since the dataset employed in the present study is different from that used by University of Michigan group, it may be possible to provide independent information by application of the technique of the present study. We plan to start such an attempt as the next step of the present study.

Acknowledgments. We thank Yuji Yagi for valuable discussions on this study. We are grateful for Nozomu Takeuchi for providing us with his DSM codes. We would like to appreciate Robert J. Geller and an anonymous reviewer, whose comments and suggestions greatly improved the manuscript.

\section{References}

Cummins, P. R., R. J. Geller, T. Hatori, and N. Takeuchi, DSM complete synthetic seismograms: SH, spherically symmetric, case, Geophys. Res. Lett., 21, 533-536, 1994.

Dziewonski, A. M. and D. L. Anderson, Preliminary reference Earth model, Phys. Earth planet. Inter., 25, 297-356, 1981.

Dziewonski, A. M. and J. H. Woodhouse, An experiment in systematic study of global seismicity: Centroid-moment tensor solutions for 201 moderate and large earthquakes of 1981, J. Geophys. Res., 88, 3247-3271, 1983.

Hara, T., CMT solution determined by Direct Solution Method, http:// iisee.kenken.go.jp/staff/thara/kushiro.htm, 2003.

Riedesel, M. A., T. H. Jordan, A. F. Sheehan, and P. G. Silver, Momenttensor spectra of the 19 Sept 85 and 21 Sept 85 Michoacan, Mexico, earthquakes, Geophys. Res. Lett., 13, 609-612, 1986.

Ruff, L. J. and A. D. Miller, Rupture process of large earthquakes in the northern Mexico subduction zone, Pure and Appl. Geophys., 142, 101$171,1994$.

Takeuchi, N., R. J. Geller, and P. R. Cummins, Highly accurate P-SV complete synthetic seismograms using modified DSM operators, Geophys. Res. Lett., 23, 1175-1178, 1996.

Tanioka, Y. and L. J. Ruff, Source Time Functions, Seism. Res. Lett., 68, 386-397, 1997.

Yagi, Y., Rupture Process for September 262003 Tokachi-Oki, Japan, earthquake, http://iisee.kenken.go.jp/staff/yagi/eq/Japan20030926/ japan20030926.html, 2003.

Yamanaka, Y. and M. Kikuchi, Source process of the recurrent Tokachioki earthquake on September 26, 2003, inferred from teleseismic body waves, Earth Planets Space, 55, e21-e24, 2003.

T. Hara (e-mail: thara@kenken.go.jp) 